\title{
Role of the expansin and xyloglucan endotransglycosylase genes in the regulation of tobacco growth under the influence of abiotic stress factors
}

\author{
B.R. Kuluev ${ }^{1,2 *}$, Kh.G. Musin ${ }^{1,2}$, Z.A. Berezhneva ${ }^{1}$, E.V. Mikhaylova ${ }^{1}$, E.A. Zaikina ${ }^{1}$ \\ 1 Institute of Biochemistry and Genetics - Subdivision of the Ufa Federal Research Centre of the Russian Academy of Sciences, Ufa, Russia \\ ${ }^{2}$ Bashkir State University, Ufa, Russia
}

DOI 10.18699/ICG-PlantGen2019-43

(c) Autors, 2019

* e-mail:kuluev@bk.ru

\begin{abstract}
The divergence of cellulose microfibrils during cell expansion is achieved by three basic mechanisms: hydrolysis of a part of binding glycans with endoglycanases, cutting and new crosslinking of glycans with xyloglucan endo-transglycosylases/hydrolases (XTHs), and the breaking of hydrogen bonds between the microfibrils of cellulose and glycan chains, which is carried out by expansins. We have created transgenic tobacco plants with the genes encoding tobacco expansins: NtEXPA1, NtEXPA4, NtEXPA5 and a gene that encodes tobacco xyloglucan endotransglycosylase, NtEXGT. Increased expression of the NtEXPA 1 and NtEXPA5 expansin genes led to an increase in growth rate and root length both under normal conditions and at $12{ }^{\circ} \mathrm{C}$ and $50 \mathrm{mM} \mathrm{NaCl}$. Increased expression of the expansin genes influenced changes in the fresh and dry mass of a shoot, leading to an increase in their exposure to hypothermia. Overexpression of the NtEXGT gene promoted tobacco root growth in a medium containing $50 \mathrm{mM} \mathrm{NaCl}$. In wild-type tobacco plants, transcript levels of the NtEXPA1, NtEXPA4, NtEXPA5, and NtEXGT genes were increased in response to abiotic stress treatment. Overall, data obtained may indicate the involvement of the NtEXPA1, NtEXPA4, NtEXPA5, and NtEXGT genes in the regulation of tobacco growth under hypothermia, drought, and salinity.
\end{abstract}

Key words: drought tolerance; expansin; xyloglucan; endotransglycosylase genes; N. tabacum

\section{Introduction}

Expansins are a group of proteins that are capable to modify the mechanical properties of plant cell walls by a nonenzymatic mechanism. These proteins are encoded by large multigene families and are widely distributed in plant species (Kuluev et al., 2016). Expansins are implicated in the growth responses of plants to adverse environments (Gao et al., 2010; Han et al., 2012; Lu et al., 2013). The enzymes of the xyloglucan endotransglycosylase family can potentially have two distinct catalytic activities, with radically different effects on xyloglucan: xyloglucan endo-transglycosylase (XET) activity results in the nonhydrolytic cleavage and ligation of xyloglucan chains, whereas xyloglucan endo-hydrolase (XEH) activity yields irreversible chain shortening (Rose et al., 2002). In angiosperms, XET/XTHs are typically encoded by large multigene families (Rose et al., 2002). There is information about the participation of XET/XTHs in providing tolerance to water deficit caused by drought and salinity (Choi et al., 2011). It has been shown that XET/XTHs expression has been induced by a broad spectrum of abiotic stresses, including drought, high salinity, and cold temperature (Kuluev et al., 2017). The aim of our study was to elucidate the molecular mechanisms of expression regulation of expansins and xyloglucan endotransglycosylases under changing environmental conditions and to determine the role of these proteins in ensuring growth and productivity, both under normal conditions and under the action of drought and hypothermia. Tobacco was chosen as a model object, including the transgenic lines of this plant expressing expansins and XTHs genes.

\section{Materials and methods}

Plants of the N. tabacum cv. 'Petit Havana SR1' were grown in 450-ml pots filled with universal soil substrate "Terra vita" in a greenhouse at $+27{ }^{\circ} \mathrm{C}$ under $140 \mathrm{mmol} \mathrm{m} \mathrm{m}^{-1}$ photon flux density and photoperiod of 16/8 hours (day/night) within 40 days (twelve leaves stage) and then subjected to stress treatments. Cadmium stress was induced by exposing plants to $100 \mu \mathrm{M}$ cadmium supplied as $\mathrm{Cd}\left(\mathrm{CH}_{3} \mathrm{COO}\right)_{2}$ for $16 \mathrm{~h}$. For freezing and cold treatments, plants were exposed to a temperature of 0 or $10^{\circ} \mathrm{C}$ for $6 \mathrm{~h}$ and $8 \mathrm{~h}$, respectively. Heat stress treatment was performed by incubating tobacco plants at $42{ }^{\circ} \mathrm{C}$ for $6 \mathrm{~h}$. Drought stress was induced by withholding water supply for 2 days. For salt stress treatment, tobacco plants were germinated on universal soil for 40 days, then roots were carefully washed and plants were transferred for 5 days on hydroponics solution (10\% Hoagland-Arnon solution). After that, plants were incubated for $2 \mathrm{~h}$ at different concentrations of $\mathrm{NaCl}(20,80,170,250,350$, and $850 \mathrm{mM})$. After stress treatments, leaves were frozen in liquid nitrogen and used to isolate total RNA. Untreated plants served as controls. Expansin genes were used as target genes: NtEXPA1, NtEXPA4, NtEXPA5 and NtEXPA6 of tobacco, as well as the $N t E X G T$ gene encoding one of the xyloglucan endotransglycosylases of tobacco. When conducting our research, we also used $\mathrm{T}_{1}$ lines $35 S: \because N t E X P A 5, D M V:: N t E X P A 1, N t E X P A 4 i$ (NtEXPA4-silenced), XVE::NtEXPA1, 35S::NtEXGT of the previously created transgenic tobacco Nicotiana tabacum L. var. 'Petit Havana SR1'. 


\section{Results and discussion}

Transcript levels of the NtEXPA1, NtEXPA4, and NtEXPA5 genes were increased in response to $\mathrm{NaCl}$, drought, cold, heat, and $10 \mu \mathrm{M}$ abscisic acid (ABA) treatments but were reduced in response to more severe stresses, i. e. cadmium, freezing, and $100 \mu \mathrm{M}$ ABA. In contrast, no changes were found in NtEXPA6 transcript level after all stress treatments. The NtEXGT gene was also up-regulated by salinity, drought, cold, cadmium and $10 \mu \mathrm{M}$ abscisic acid treatments and down-regulated in response to $0{ }^{\circ} \mathrm{C}$ and $100 \mu \mathrm{M}$ abscisic acid. Based on the data obtained, it was possible to suggest that the genes studied are involved in the regulation and maintenance of growth under the action of abiotic stress factors. In addition, we examined the involvement of tobacco expansins and XTHs in the regulation of abiotic stress tolerance by transgenic approaches. Transgenic tobacco plants with constitutive expression of NtEXPA1 and NtEXPA5 exhibited improved tolerance to salt stress: these plants showed higher growth indices after $\mathrm{NaCl}$ treatment and minimized water loss by reducing stomatal density. In contrast, NtEXPA4-silenced plants were characterized by a considerable growth reduction under salinity and enhanced water loss. Root growth and stress tolerance of transgenic tobacco plants with constitutive expression of the NtEXPA1 and NtEXPA5 genes, as well as with reduced expression of the NtEXPA4 gene with prolonged cultivation under drought, salinity and low positive temperatures, were investigated. Increased expression of expansin genes led to an increase in the growth rate and the length of the roots, both under normal growing conditions and following exposure to a temperature of $+12^{\circ} \mathrm{C}$ and $50 \mathrm{mM} \mathrm{NaCl}$. Also, increased expression of expansin genes influenced the change in fresh and dry weight of the shoot, leading to their increase when they were exposed to hypothermia. Transgenic plants with a reduced level of expression of the expansin NtEXPA4 gene were characterized by a decrease in the fresh and dry weight of the shoot under the action of drought and low positive temperatures. $35 S: \because N t E X G T$ tobacco plants also showed higher rates of root growth under salt-stress conditions, greater frost and heat tolerance as compared with the wild-type tobacco plants.

\section{Conclusions}

Overall, data obtain may indicate the involvement of the NtEXPA1, NtEXPA4, NtEXPA5 and NtEXGT genes of tobacco in growth regulation under the action of hypothermia, drought and salinization. We suppose that genes can be used to improve root growth, as well as to increase the productivity of transgenic plants under the action of hypothermia and moderate salinity.

\section{References}

Choi J.Y., Seo Y.S., Kim S.J., Kim W.T., Shin J.S. Constitutive expression of CaXTH3, a hot pepper xyloglucan endotransglycosylase/hydrolase, enhanced tolerance to salt and drought stresses without phenotypic defects in tomato plants (Solanum lycopersicum cv. Dotaerang). Plant Cell Rep. 2011;30:867-877. DOI 10.1007/s00299-010-0989-3

Gao X., Liu K., Lu Y.T. Specific roles of AtEXPA1 in plant growth and stress adaptation. Russ. J. Plant Physiol. 2010;57:241-246. DOI 10.1134/ S1021443710020111.

Han Y., Li A., Li F., Zhao M., Wang W. Characterization of a wheat (Triticum aestivum) expansin gene, TaEXPB23, involved in the abiotic stress response and phytohormone regulation. Plant Physiol. Biochem. 2012;54:49-58. DOI 10.1016/j.plaphy.2012.02.007.

Kuluev B.R., Avalbaev A.M., Mikhaylova E.V., Nikonorov Y.M., Berezhneva Z.A., Chemeris A.V. Expression profiles and hormonal regulation of tobacco expansin genes and their involvement in abiotic stress response. J. Plant Physiol. 2016;206:1-12. DOI 10.1016/j.jplph.2016.09.001.

Kuluev B.R., Mikhaylova E.V., Berezhneva Z.A., Nikonorov Y.M., Postrigan B.N., Kudoyarova G.R., Chemeris A.V. Expression profiles and hormonal regulation of tobacco NtEXGT gene and its involvement in abiotic stress response. Plant Physiol. Biochem. 2017;111:203-215. DOI 10.1016/j.plaphy.2016.12.005.

Lu P., Kang M., Jiang X., Dai F., Gao J., Zhang C. RhEXPA4, a rose expansin gene, modulates leaf growth and confers drought and salt tolerance to Arabidopsis. Planta. 2013;237:1547-1559. DOI 10.1007/s00425-0131867-3.

Rose J.K.C., Braam J., Fry S.C., Nishitani K. The XTH family of enzymes involved in xyloglucan endotransglucosylation and endohydrolysis: current perspectives and a new unifying nomenclature. Plant Cell Physiol. 2002;43:1421-1435. DOI 10.1093/pcp/pcf171.

Acknowledgements. The study was performed within the framework of the State Assignment (project no. AAAA-A19-119021190011-0) and also supported by Russian Foundation for Basic Research grant No. 18-04-00118A.

Conflict of interest. The authors declare no conflict of interest. 